\title{
An intein with genetically selectable markers provides a new approach to internally label proteins with GFP
}

\author{
Richard Ramsden, Luther Arms, Trisha N Davis and Eric GD Muller
}

\begin{abstract}
Background: Inteins are proteins that catalyze their own removal from within larger precursor proteins. In the process they splice the flanking protein sequences, termed the $\mathrm{N}$-and $\mathrm{C}$-terminal exteins. Large inteins frequently have a homing endonuclease that is involved in maintaining the intein in the host. Splicing and nuclease activity are independent and distinct domains in the folded structure. We show here that other biochemical activities can be incorporated into an intein in place of the endonuclease without affecting splicing and that these activities can provide genetic selection for the intein. We have coupled such a genetically marked intein with GFP as the Nterminal extein to create a cassette to introduce GFP within the interior of a targeted protein.

Results: The Pch PRP8 mini-intein of Penicillium chrysogenum was modified to include: 1) aminoglycoside phosphotransferase; 2) imidazoleglycerol-phosphate dehydratase, His5 from S. pombe; 3) hygromycin B phosphotransferase; and 4) the transcriptional activator LexA-VP16. The proteins were inserted at the site of the lost endonuclease. When expressed in E. coli, all of the modified inteins spliced at high efficiency. Splicing efficiency was also greater than $96 \%$ when expressed from a plasmid in S. cerevisiae. In addition the inteins conferred either G418 or hygromycin resistance, or histidine or leucine prototropy, depending on the inserted marker and the yeast genetic background. DNA encoding the marked inteins coupled to GFP as the N-terminal extein was PCR amplified with ends homologous to an internal site in the yeast calmodulin gene CMD1. The DNA was transformed into yeast and integrants obtained by direct selection for the intein's marker. The His5-marked intein yielded a fully functional calmodulin that was tagged with GFP within its central linker.

Conclusions: Inteins continue to show their flexibility as tools in molecular biology. The Pch PRP8 intein can successfully tolerate a variety of genetic markers and still retain high splicing efficiency. We have shown that a genetically marked intein can be used to insert GFP in one-step within a target protein in vivo.
\end{abstract}

\section{Background}

Inteins are small proteins naturally found either embedded within a larger precursor protein (cis-acting inteins), or split between two pro-proteins (trans-acting inteins). They have the unique ability to self-catalyze their excision from the pro-protein(s) and in the process form a peptide bond between the flanking amino acid sequences that reside at the $\mathrm{N}$-and $\mathrm{C}$-terminal boundaries with the intein $[1,2]$. These flanking regions adjacent to the intein are referred to as exteins, analogous to the terminology for introns and exons. An intein acts

\footnotetext{
* Correspondence: emuller@uw.edu Department of Biochemistry, University of Washington, Seattle, WA, USA
}

autonomously, splicing together the exteins without the need of a co-factor or an assisting complex. Since splicing is post-translational, for cis-acting inteins a single open reading frame driven by a single promoter expresses two distinct mature protein products: the spliced exteins and the intein.

The first intein described, Sce VMA1, was found in a subunit of the vacuolar $\mathrm{H}^{+}$-adenosine triphosphatase complex of the budding yeast Saccharomyces cerevisiae $[3,4]$. Currently over 500 inteins are known, found in over 200 species of microorganisms in over 70 different proteins [5]. Present in all three phylogenetic domains of life, inteins in Eukaryotes are predominantly found in fungi and unicellular alga [5].

\section{Biomed Central}


A homing endonuclease is often present within cis-acting inteins. The splicing and endonuclease domains are structurally and functionally distinct, as shown both by the crystal structure of Sce VMA1 [6] and the conservation of splicing following the deletion of the homing endonuclease [1,7]. In addition minimal or mini-inteins lacking the homing endonuclease occur naturally or have been engineered to be as small as 143 amino acids $[8,9]$.

The use of inteins in molecular biology, biotechnology and drug discovery has recently been reviewed [10-12]. The broad spectrum of applications for inteins is in part due to the minimal constraints placed on the sequence of the exteins. The only absolute requirement is the presence of cysteine, serine or threonine at the downstream splice junction in the C-terminal extein. There are no known size constraints. Splicing efficiency is reduced if the extein domains are unfolded or have poor solubility, but in vitro even this can usually be overcome with detergents or refolding regimes [13]. The immediate flanking sequence of the intein can affect splicing efficiency to varying degrees [14], but a variety of sequences are tolerated. Thus the inherent tolerance of inteins allows them to be adapted to splice proteins of diverse character.

In this study the flexibility of inteins has been exploited to investigate the potential for inteins to direct in vivo gene modification, specifically the tagging of an internal region of a protein with GFP. The microscopy group of the Yeast Resource Center is focused on using Förster resonance energy transfer, FRET, to study the organization of large protein complexes in vivo [15-18]. A limiting step for FRET studies is identifying regions within proteins that can accept a GFP-related fluorescent protein tag. As a first step towards overcoming this limitation, we engineered genetic markers within the Pch PRP8 intein that provided a way to internally tag proteins in one step in yeast. The Pch PRP8 intein was chosen for its robust, temperature-independent splicing activity $[9,19]$ and the engineered inteins continued to display very high splicing efficiency.

\section{Results}

\section{Pch PRP8 intein splices with inserts at the empty} endonuclease site

During the course of their initial characterization of the Pch PRP8 mini-intein Elleuche et al. [19] aligned the sequence of the intein with PRP8 inteins from other species. The position of a presumptive lost homing endonuclease was identified from a gap in the alignment. This position, designated "E", later was shown to tolerate a deletion of six amino acids without loss of splicing activity [9]. In addition the intein could be split in half at this position and the resulting two independent proteins, when expressed in E. coli, had active trans-splicing activity [20].

Given the tolerance of the "E" position for change, this site in the Pch PRP8 intein was chosen for the insertion of genes encoding genetically selectable enzymes. These enzymes were: 1) aminoglycoside phosphotransferase; 2 ) imidazoleglycerol-phosphate dehydratase from S. pombe; and 3) hygromycin B phosphotransferase. They confer G418/kanamycin resistance, histidine prototrophy and hygromycin resistance in the appropriate yeast genetic background, respectively. In addition the gene encoding the transcriptional activator LexA-VP16 [21] was inserted. These proteins share in common a modest size (216, 269, 280 and 341 amino acids for His5, G418 ${ }^{\mathrm{R}}$, LexA-VP16 and Hyg $^{\mathrm{R}}$, respectively) all below the typical size of homing endonucleases (350-450 aa). Perhaps more importantly the $\mathrm{N}$-and $\mathrm{C}$-terminal ends of each protein are proximal to each other [22-25]. A schematic diagram of these constructs is shown in Figure 1.

We used the experimental system of Elleuche et al. [19] to test whether the Pch PRP8 intein with inserted markers could splice. In their system GST is the $\mathrm{N}$-terminal extein and a $6 \times$ His-tag is the $\mathrm{C}$-terminal extein. The embedded intein is expressed in E. coli and splicing results in the production of GST- $6 \times$ His and the released intein.

We confirmed the robust splicing activity of Pch PRP8 intein (Figure 2, lane 2). GST-6 $\times$ His and the released intein are clearly visible in SDS-PAGE, and the identity of spliced GST- $6 \times$ His was confirmed by Western blot analysis with anti- $6 \times$ His and anti-GST antibodies. The addition of cloning sites flanked by flexible protein linkers at position $\mathrm{E}$ did not impair splicing since the free inteins and the GST- $6 \times$ His were clearly visible in SDS-PAGE with no evidence of unspliced products (Figure 2, lane 3).

The modified inteins with the 4 different selection marker cassettes also showed highly efficient splicing

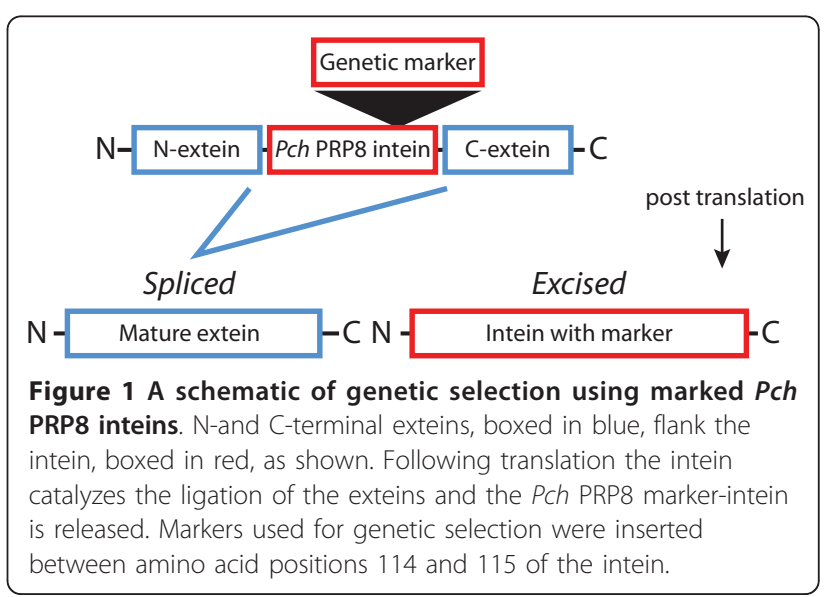




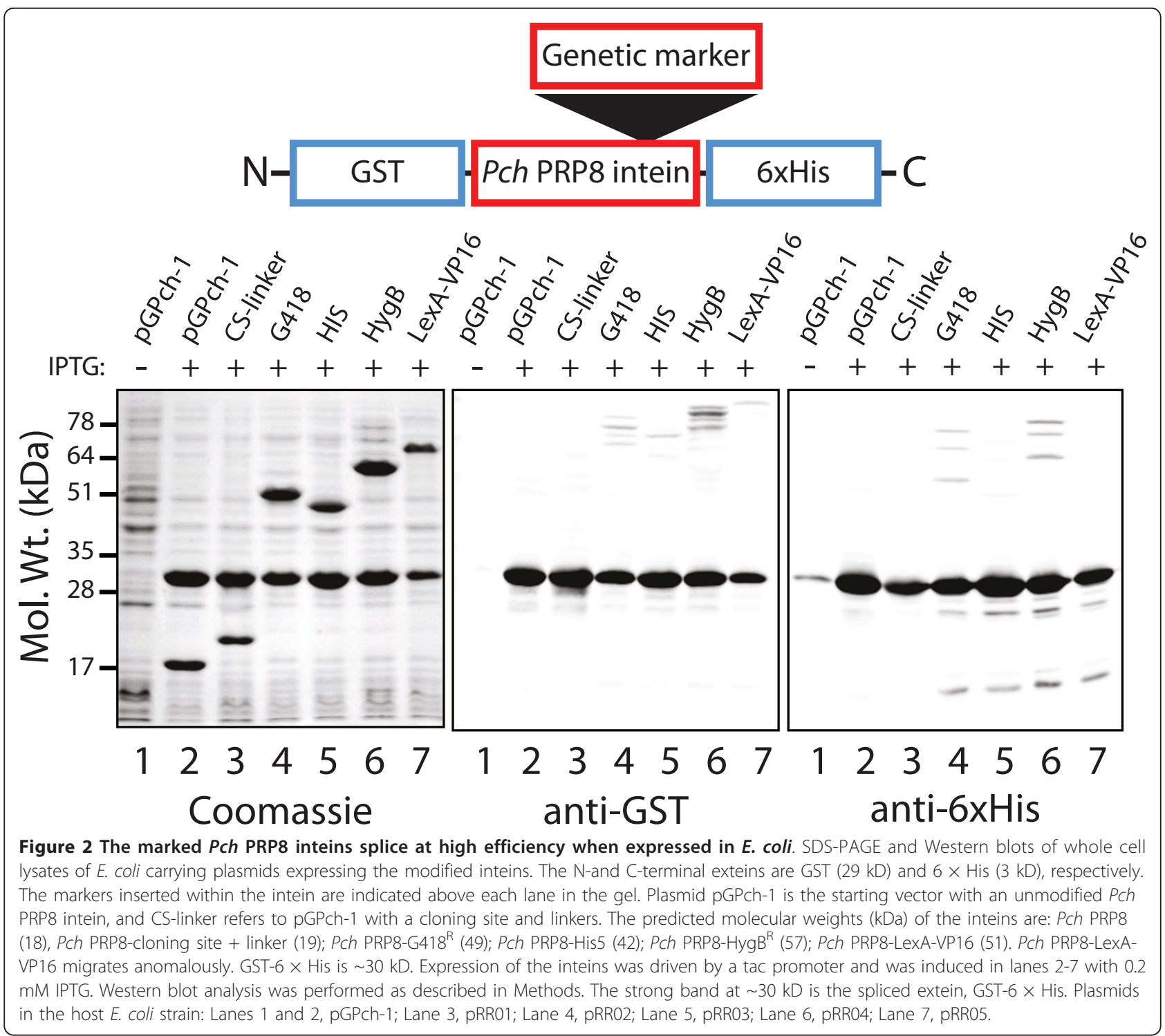

(Figure 2, lanes 4-7). Based on the ratio of spliced products to larger, unspliced proteins in the Western blots, the splicing efficiencies ranged from $\sim 88 \%$ for the Pch PRP 8 Hyg $^{\mathrm{R}}$-intein to almost $100 \%$ for the His5-and LexA-VP16-inteins. Thus the Pch PRP8 intein tolerated the insertion of four different proteins at the vacant endonuclease site, maintaining nearly complete excision and splicing activity in this system.

The marked Pch PRP8 inteins splice in yeast and confer selectable genetic traits

To determine whether the modified inteins would function in yeast we constructed a new panel of yeast CEN4/URA3 plasmids that contained the marked inteins expressed under the control of the GAL1 promoter. For these plasmids the $\mathrm{N}$-terminal extein was GFP containing a nuclear localization signal (NLS) and the C-terminal extein was the FLAG peptide, DYKDDDDK. Just as in the previous E. coli plasmids, the extein/intein junction encodes five $\mathrm{N}$-and four C-terminal extein residues of the P. chrysogenum Prp8 protein. The spliced product is NLS-GFP-FLAG (33 $\mathrm{kD})$ and the released intein carries the selectable genetic marker or a transcription factor. NLS-GFP provided a simple way to follow expression and proper folding of the extein.

In agreement with the results in E. coli, all of the modified inteins when expressed in yeast displayed high efficiency splicing (Figure 3). Based on the limits of detection in the anti-GFP Westerns, we estimate that the efficiency of splicing is greater than $96 \%$ for the modified inteins. 


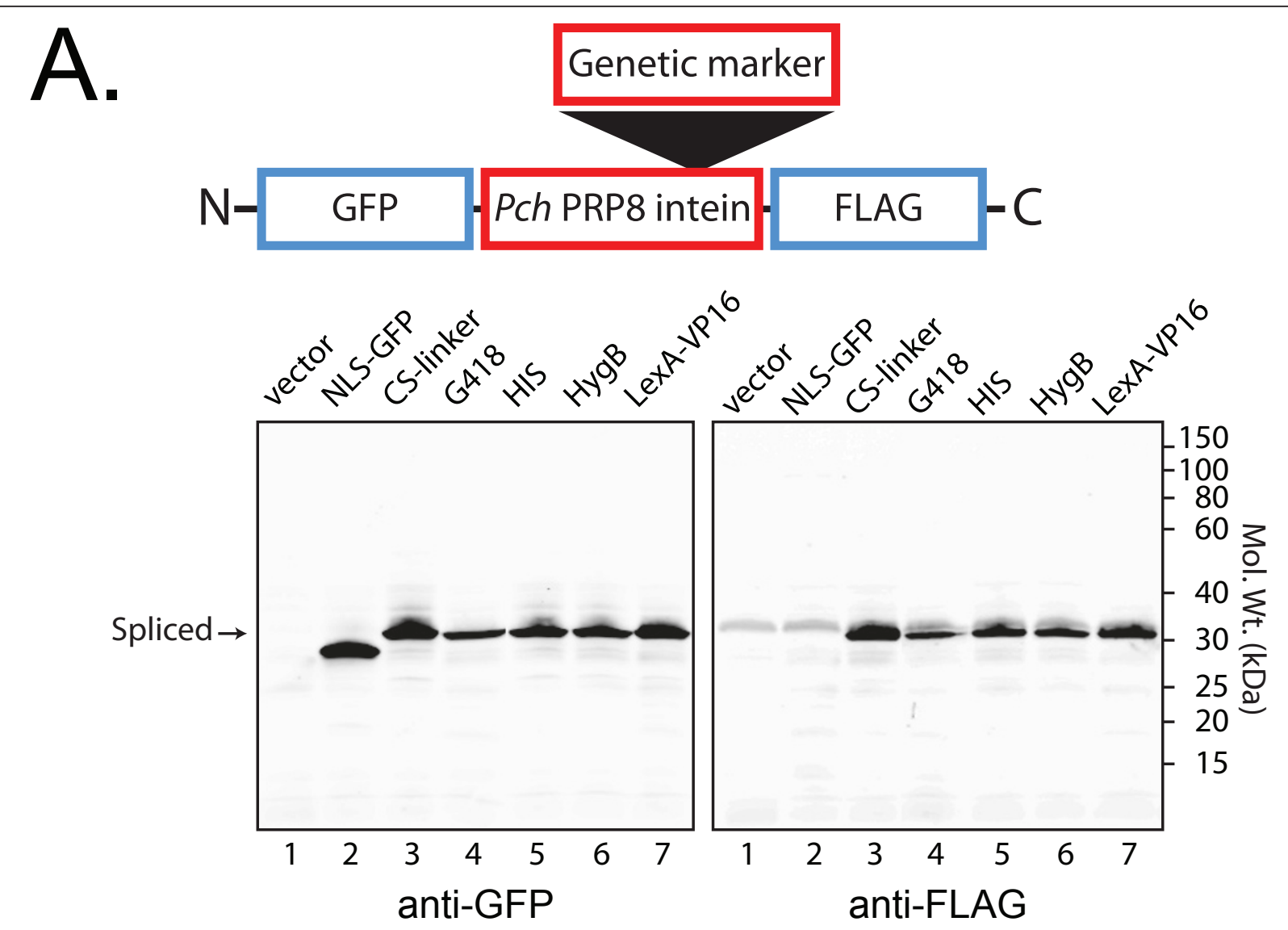

B.

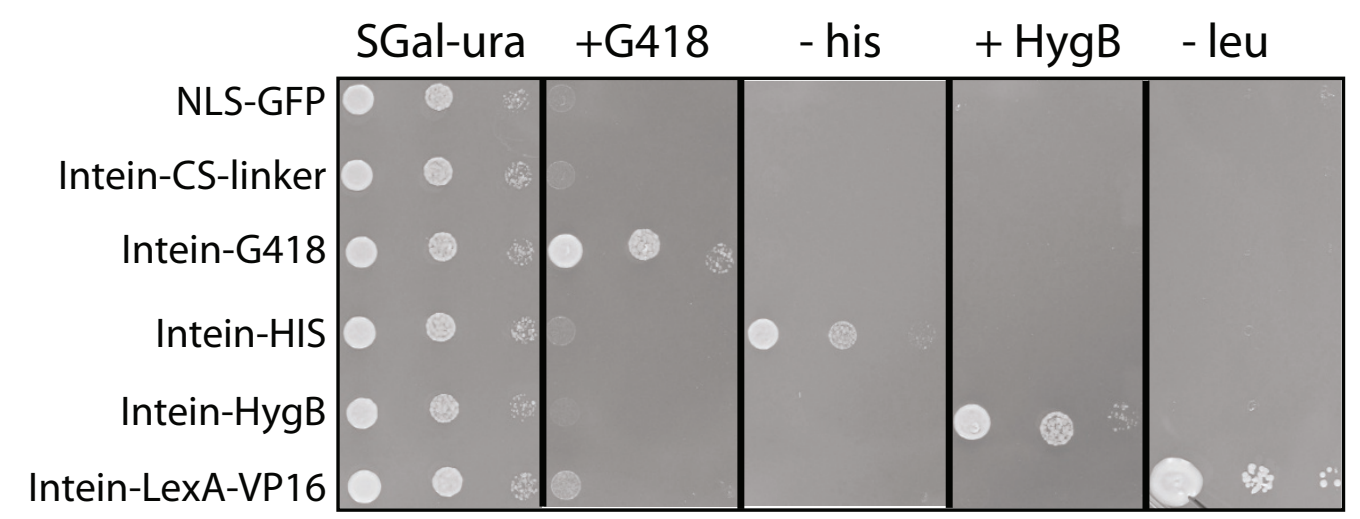

Figure 3 Marked Pch PRP8 inteins splice and allow genetic selection in yeast. The inteins described in Figure 2, but with different exteins, were expressed in yeast under the control of the GAL1 promoter. For expression in yeast, the N-extein was GFP with a nuclear localization signal, and the C-extein was a FLAG epitope tag. The spliced extein product is NLS-GFP-FLAG (33 kD). The molecular weights of the Pch markerinteins are given in Figure 2. However in the anti-GFP and anti-FLAG Westerns there is no clear evidence for unspliced or partially spliced products of higher molecular weight than the spliced product, such as seen in Figure 2. A. Western blots showing the high efficiency of splicing in strain YL $(2,4)$ LU expressing the modified inteins (and controls) from the following plasmids: Lane 1, pBM258; Lane 2, pRR26; Lane 3, pRR21; Lane 4, pRR22; Lane 5, pRR23; Lane 6, pRR24; Lane 7, pRR25. Analysis was performed as described in Methods. The genetic markers of the inteins and antibodies used for Western blots are indicated above and below the gels, respectively. Note that in the anti-FLAG western a background band was apparent in all lanes, including the protein extracts from the vector alone and NLS-GFP expression in lanes 1 and 2. B. Five-fold serial dilutions of the various yeast strains were prepared and spotted onto SGal-ura medium, SGal-ura medium lacking histidine or leucine, or SGal-ura supplemented with antibiotic as indicated. The plasmid backbone confers uracil prototrophy. 
Concomitant with splicing the intein is released from the precursor protein. The inteins did not inhibit growth and provided resistance to G418 or hygromycin in cells expressing the Pch PRP8 G418 ${ }^{\mathrm{R}}$-intein or the $\mathrm{HygB}^{\mathrm{R}}$ intein, respectively (Figure 3B). Likewise the Pch PRP8 His5-intein was able to complement the mutation in HIS3 in this genetic background (Figure 3B). Finally the Pch PRP8 LexA-VP16-intein was able to drive expression of a LEU2 gene that had $2 \operatorname{lexA}$ operator sequences within its promoter and confer selectable leucine prototrophy to the yeast strain. Thus in all cases each marker remained functional within the context of the excised intein.

\section{Direct integration of the marked inteins at the CMD1 locus}

Having established that the intein remained active and the embedded markers functional in yeast, we next examined whether the marked inteins could provide a method to introduce GFP within the coding sequence of a targeted gene. For our test we chose $C M D 1$, the gene encoding yeast calmodulin [26]. Calmodulin contains two globular EF-hand calcium-binding domains separated by a flexible linker. The design of the experiment is presented in Figure 4. Briefly the GFP-Pch PRP8 marker-intein cassette was PCR amplified using oligonucleotide primers with ends homologous to the center of the

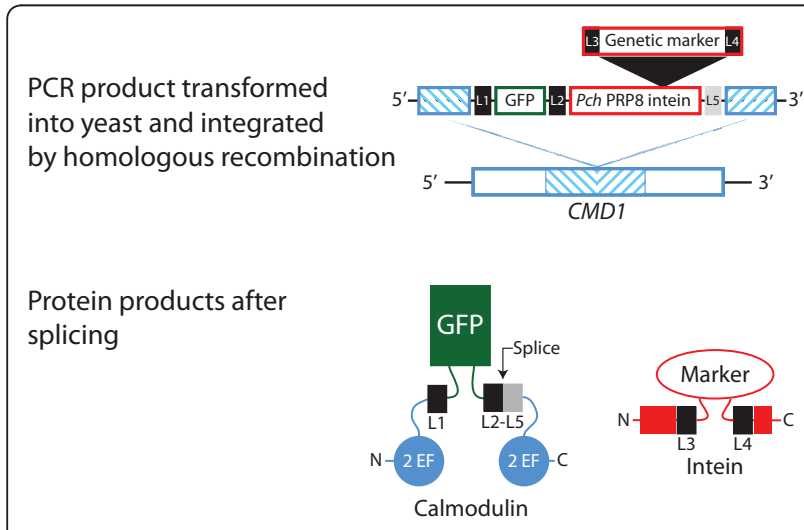

Figure 4 Schematic showing the experimental design to integrate the marked Pch PRP8 inteins at the CMD1 locus. The top panel shows the composition and organization of the PCR amplified DNA used to transform yeast. The blue hatched area corresponds to the central sequence of the CMD1 gene that was the target for homologous recombination. L1-L5 are linker regions. In the bottom panel are the two proteins produced after splicing: 1) calmodulin with GFP inserted between $\mathrm{S} 79$ and N80, and 2) Pch PRP8 intein with genetic markers inserted at the vacant endonuclease site. The junction between $L 2$ and $L 5$ is the splice site. L2 and L5 contain 5 and 4 amino acids from the original Pch Prp8 extein sequences. For a description of the linker sequences see Methods. EF refers to the calcium-binding EF-hand domains. The sizes of the regions are drawn for clarity and are not to scale.
CMD1 gene encoding the central linker region. Transformation and direct selection for the markers within the inteins isolates cells in which the Pch PRP8 GFPintein cassettes were inserted by homologous recombination between amino acids 79 and 80 of calmodulin.

Transformation with DNA from the Pch PRP8 G418 ${ }^{\mathrm{R}}$ , His5-and $\operatorname{HygB}^{\mathrm{R}}$-inteins produced transformants with the selected auxotrophic or antibiotic resistance, while the Pch PRP8 LexA-VP16-intein did not yield transformants. Since the level of expression of the intein is dependent on the level of CMD1 expression we conclude that the LexA-VP16 was not at a level to fully activate lexAop ${ }_{6}$ LEU2 gene expression in strain YL $[6,4]$ LU. However in agreement with the previous results from yeast episomal expression, the recovery of resistant or prototrophic cells from the other Pch PRP8 markedinteins again showed that the intein domain did not impair the activity of the selection markers.

In our experimental design the intein was initially transformed into a diploid strain so that transformants could be recovered even if the integration of the intein impaired the expression or activity of calmodulin. Diploid transformants were sporulated and the tetrads dissected to yield four haploids, two with the intein insertion and two without. The results of the tetrad analysis are shown in Figure 5A. The Pch PRP8 His5-intein $C M D 1$ insertions grew at the same rate as wild-type, but both the Pch PRP8 G418 ${ }^{\mathrm{R}}$-and $\mathrm{HygB}^{\mathrm{R}}$-inteins impaired growth.

Associated with the impaired growth was evidence of inefficient splicing (Figure 5B). The Pch PRP8 His5intein was effectively spliced from calmodulin, producing Cmd1-GFP at high efficiency (lane 3). On the other hand, the $G 418^{\mathrm{R}}$-intein (lanes 4,5) and $\mathrm{HygB}^{\mathrm{R}}$-intein (not shown) were not. Different isolates showed varying levels of accumulation of unspliced product (lanes 4 and $5)$. The slow growth and phenotypic variation of the G418 ${ }^{\mathrm{R}}$ and $\mathrm{Hyg}^{\mathrm{R}}$ isolates precluded further analysis and in any case meant that these inteins were not suitable for tagging Cmd1.

Calmodulin concentrates at sites of cell growth and at the spindle pole body in yeast [27-29]. The wild-type localization pattern was seen for Cmd1 with the internal GFP-His5-intein (Figure 5C). In agreement with the published results, unbudded cells had calmodulin in puncta at the cell membrane. In newly budded cells calmodulin formed a patch at the bud tip that dispersed as the bud grew. Preceding cytokinesis calmodulin was concentrated at the bud neck. Localization at the pair of spindle pole bodies is best seen in the medium budded cell in Figure 5. For comparison the online Yeast Resource Center Public Image Repository [29] contains over 2000 images of yeast showing the localization of CMD1 tagged with different fluorescent proteins. 

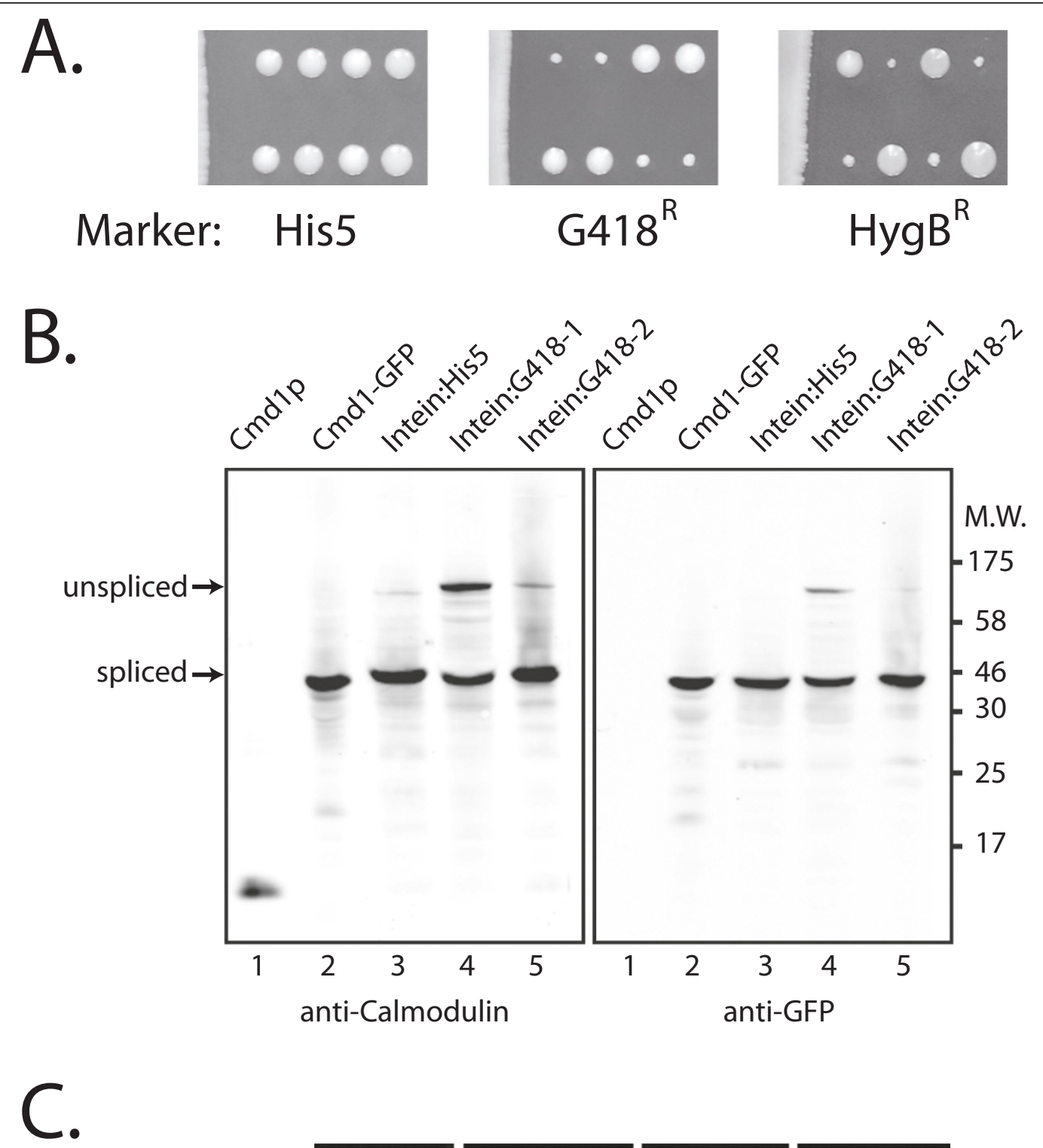

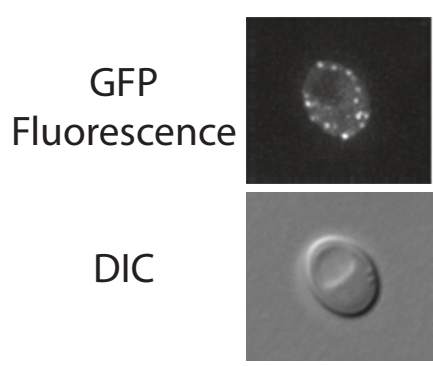

1
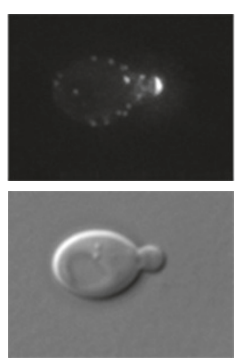

2
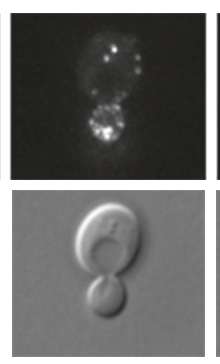

3
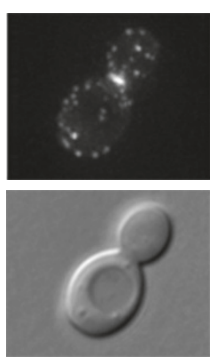

4

Figure 5 Integration of the marked Pch PRP8 inteins at CMD1 locus. A. Tetrad analysis of diploids that are heterozygous for the insertion of the marked inteins at the CMD1 locus (CMD1/CMD1::GFP-PCh PRP8 marker-intein). Individual haploid spores were grown on non-selective YPD medium. Segregation of the mutant alleles was 2:2 and the slow growing colonies contain CMD1 with the G418 ${ }^{R}$-and HygB ${ }^{R}$-intein insertions as determined by plating the haploids on selective medium (not shown). B. Western blot analysis of protein extracts from haploids containing the PCh PRP8 marked-inteins at the CMD1 locus. Lane 1, 100 ng purified calmodulin; Lane 2 LAY18-2C expressing Cmd1-GFP (constructed using the classic PCR method [41]); Lane 3, extract from a haploid CMD1::GFP-PCh PRP8-HIS5-intein isolate; Lanes 4, 5 extracts from two separate haploid CMD1::GFP-PCh PRP8-G418 ${ }^{R}$-intein isolates. C. Fluorescence microscopy of the haploid CMD1::GFP-PCh PRP8-HIS5-intein isolate. Numbers 1-4 refer to images showing unbudded, small budded, medium budded or large budded cells, respectively. 


\section{Discussion}

Inteins are widely used in biotechnology to manipulate proteins. The capacity of inteins to break and form peptide bonds has been exploited to develop a range of methodologies from a routine procedure to purify proteins, to more advanced semi-synthetic applications that assemble proteins for NMR, crystallography and mechanistic studies. These uses and others have been recently reviewed $[10,11,30]$.

The use of inteins as genetic tools is still an emerging area of research. Temperature-sensitive inteins [31-33], light-activated inteins [34], and inteins controlled by small molecules [35-37], provide a way to control protein activity by controlling when a protein that has been interrupted by an intein is spliced together to yield its mature form. Conditional protein assembly by inteins in vivo is a promising method to generate temperaturesensitive mutants, and target when and where a protein is functional in a cell.

Here we add a new role for inteins in genetic manipulations, as a genetic marker with the unique property of self-excision. The engineered intein marks the expression of the protein into which it is embedded and yet after splicing is not a part of the mature protein. We demonstrate in yeast the use of a Pch PRP8 His5marked intein to deliver, in one step and in vivo, GFP within a targeted protein without disturbing the function of the protein. In principle, the method could be extended to other protein tags, and adapted to other organisms.

We created a family of genetically marked inteins based on the PRP8 intein of Penicillium chrysogenum. PRP8 inteins have an ancient heritage and are widespread [19,38-40]. However they are not found in S. cerevisiae. The Pch PRP8 intein and the native yeast VMA1 intein are sufficiently divergent (21\% sequence identity) to preclude homologous recombination between the two during the transformation step. For comparison the $S$. cerevisiae HIS3 and the S. pombe his $5^{+}$genes share $58 \%$ sequence identity and yet do not recombine during integrative transformation [41]. The sequence divergence, the fact that the Pch PRP8 intein is well characterized $[9,19,20,39]$, splices at high efficiency across a broad range of temperatures [19] and lacks a DNA binding domain led to its choice in our studies.

The primary sequence of the Pch PRP8 mini-intein shares sequence similarity with other inteins [20]. Thus its three-dimensional structure likely conforms to the canonical Hedgehog/Intein (HINT) protein scaffold [42-48]. The HINT module has a flat horseshoe shape with a series of $\beta$-strands forming the framework. The active-site is centered at the interior base or "toe" of the horseshoe. Endonucleases if present in the intein are a separate domain located away from the active site region at the "heel" of the horseshoe.

Here we show that the Pch PRP 8 intein, and presumably other inteins, have a remarkable capacity to accept foreign protein domains at the region of the structure where the homing endonuclease resides in full inteins. High splicing efficiency was observed for all of the inserted single domain proteins that were used for genetic markers. We attribute the success to several factors. An alignment of the Pch PRP8 intein with the Synechocystis sp. DnaB and the Mycobacterium xenopi GyrA inteins shows that the insertion site for our genetic markers is located in disordered loops in the crystal structures of both of these inteins [42,44]. Thus the region of insertion is inherently flexible and unstructured. In addition, one shared feature of the proteins used for markers is that their $\mathrm{N}$-and $\mathrm{C}$-termini are proximal [22-25]. The exit of the inserted protein domain out of the intein and its return occurs nearby in space and would tend to minimize strain in the structure of the intein. Since the majority of single domain proteins have some region of their two termini within $5 \AA$ $[49,50]$ and termini are consistently found at the surface of the protein [51], we predict that many single domain proteins could be inserted into inteins without detriment to splicing efficiency.

Three enzymes and a transcription factor were used as genetic markers to modify the Pch PRP8 intein. In pilot experiments all four modified inteins showed high splicing efficiency, both in E. coli and yeast, and in yeast the proteins within the excised inteins were active and enabled growth under conditions of selection. However when these inteins were used to deliver GFP within calmodulin only the strain tagged with the Pch PRP8 His5intein grew normally. Under selective conditions transformation with the Pch PRP8 LexA-VP16-intein did not yield any transformants, and the G418 ${ }^{\mathrm{R}}$-and $\mathrm{Hyg}^{\mathrm{R}}$ inteins yielded cells with poor growth and diminished splicing efficiency. The disruption of calmodulin function was surprising, given the pilot results, and is not understood. It is possible that the unspliced products which accumulate interfere with growth. We are actively investigating ways to boost the level of stable protein and the efficiency of splicing through changes in codon usage and the composition of the linkers between the various domains. In addition, given the success of the Pch PRP8 His5-intein, we are constructing other inteins with metabolic markers that may require lower levels of expression.

Our intein based method to insert the gene encoding GFP within an ORF offers several advantages over other PCR-based methods available in yeast. Other methods [52-54] introduce a gene with its own promoter that 
must be first be removed before the GFP is correctly in frame. Thus a second recombination event is required, either catalyzed by the induction of Cre-recombinase or by a rare natural recombination event that is isolated by genetic selection, to remove the genetic marker with its associated promoter. In addition the Cre-recombinase based system leaves behind within the ORF a peptide sequence coded for by the remaining loxP site, a sequence that can disrupt activity (unpublished). The introduced gene also temporarily interrupts the expression of the target protein. With the intein-based method integration and tagging occur in one step. Since the marked inteins are inserted in frame, expression of the target is only disrupted during recombination. Given these advantages marked inteins show great promise and complement other available methods.

\section{Conclusions}

We have shown that the Pch PRP8 intein can incorporate a variety of selectable genetic markers and still exhibit high splicing activity. This led to the development of a simple method to insert GFP within the interior of a protein in yeast. This novel use of an intein has the potential to provide a way to add, substitute, or delete domains within targeted proteins in a single step in yeast. In addition it may find application in other systems as well, such as recombineering, a method that is also based on genetic manipulation through recombination.

Refinement of this method is underway at the Yeast Resource Center. Primary areas to investigate will be the effect of codon optimization, the utility of other selection markers, and cassette development for other useful tags such as the FRET partners YFP and CFP.

\section{Methods}

\section{Strains and media}

KGY315 [55] (MATa/MATo, ade2-1oc/ade2-1oc, ADE3/ ade3 100 , can1-100/can1-100, CYH2 $2^{s} / \operatorname{cyh} 2^{r}$, his3-11,15/ his3-11,15, leu2-3,112/leu2-3,112, trp1-1/trp1-1, ura3-1/ ura3-1) was derived from W303 [56]. YL(2, 4)LU

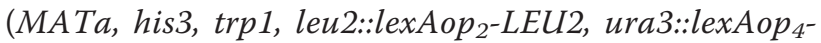
SPO13TATA-URA3) is a reporter strain for LexA-VP16 expression [21]. $\mathrm{YL}(6,4) \mathrm{LU}$ is the same as $\mathrm{YL}(2,4) \mathrm{LU}$ except that there are 6 lexAop sequences before LEU2. LAY18-2C was derived from KGY315 in which the Cterminus of CMD1 was tagged with GFP as described at the Yeast Resource Center web site http://depts. washington.edu/yeastrc/pages/fm_3.html. Escherichia coli strain TunerTM(DE3) (EMB, San Diego, CA) was used for expression and XL10-Gold ${ }^{\circledR}$ (Stratagene, La Jolla, CA) for routine cloning.

Yeast were grown in either synthetic dextrose minimal media (SD) or synthetic galactose minimal media with appropriate supplements, or yeast peptone dextrose-rich medium (YPD) with added antibiotics [17]. E. coli was grown in NZCYM medium with antibiotics.

\section{Plasmid construction}

Plasmid pGPch-1 (gift of Stefanie Pöggeler) [19] was the source of the Pch PRP8 intein and contains an $\mathrm{N}$-terminal extein of glutathione S-transferase (GST) and a C-terminal extein of a $6 \times$ His epitope tag. pRR01 is derived from pGPch-1. The $\Delta \mathrm{E}$ sequence [9], 5'-GGCGCCGATGATTCGGCT-3', coding for GAD DSA, was replaced with 5'-GGCGCCGCAG GTGCAG GTGC AGGTGCAGCT AGCGCAGCAGGTACCG CGGG TGCTGGTGCT GGTGCTTCGG CT-3' (NheI and KpnI sites underlined), to code for the protein sequence GAAGAGAGAASAAGTAGAGAGASA. Six codons within the intein sequence were optimized for expression in yeast by site-directed mutagenesis using the QuikChange ${ }^{\circledR}$ protocol (Stratagene, La Jolla, CA): Leu2 (CTC to TTG); Gly5 (GGG to GGA); Leu8 (CTC to TTG); Arg10 (CGA to AGA); Arg46 (CGC to AGA); and Arg49 (CGC to AGA). Numbering is relative to Cys (in the sequence CLAK) at position 1.

Plasmids pRR02-05 were derived from pRR1. The $G 418^{R}$ gene, the $H y g^{R}$ gene, the his $5^{+}$gene of Schizosaccharomyces pombe and the lexA-VP16 fusion were PCR amplified from pBS7, pBS4, and pDH5 (YRC, University of Washington) [57] and pBS LexA::VP16-SV40 (gift of Tzumin Lee, Janelia Farm Research Campus) [58], respectively. The ORFs were amplified with NheI and KpnI ends and inserted at the NheI/KpnI junction in pRR01. All amplifications for cloning were carried out using Vent $_{R}{ }^{\circledR}$ DNA polymerase (New England Biolabs, Beverly, MA).

Plasmids pRR11-15 and pRR21-25 include the intein sequence from the corresponding marked inteins in the pRR02-05 series. However the N-extein is either GFP or GFP with a nuclear localization signal, NLS-GFP, and the $\mathrm{C}$-extein is a $\mathrm{FLAG}^{\circledR}$ epitope. In addition protein linker sequences have been inserted at the junctions between ORFs. These linkers, L2-L5 in Figure 4, encode the following protein sequences, with adjacent sequences in parentheses: L2, (...LYK)-GAGAGAGAGAG-(FWEKACLAK...); L3, (...KGA)-AGAGAGAA(SG...); L4, AGAGAGA-(SAQ....); and L5, (SGFE)-GSG. The underlined sequences are from the $\mathrm{N}$-and $\mathrm{C}$ terminal exteins of P. chrysogenum Prp8.

As described in Table 1 these constructs were cloned into 3 base plasmids: pGEX-4T-1 (Amersham Bioscience, Europe GmbH, Freiburg, Germany); pET-Duet modified to contain only one promoter (EMD Biosciences, San Diego, CA), and pBM258 [59]. The complete DNA sequences are deposited in Genbank [60]. 


\begin{tabular}{|c|c|c|c|c|}
\hline Name & Base plasmid & Promoter & Origin/marker & Extein[intein]extein \\
\hline pGPch-1 & pGEX-4T-1 & tac & ori/Amp & GST[PCh PRP8]HIS \\
\hline pRR01 & pGEX-4T-1 & tac & ori/Amp & GST[Pch PRP8 linker] $\mathrm{HIS}_{6}$ \\
\hline pRRO2 & pGEX-4T-1 & tac & ori/Amp & GST[PCh PRP8 G418 $\left.{ }^{R}\right] \mathrm{HIS}_{6}$ \\
\hline pRR03 & pGEX-4T-1 & tac & ori/Amp & GST[Pch PRP8 HIS5]HIS \\
\hline pRR04 & pGEX-4T-1 & tac & ori/Amp & GST[Pch PRP8 HygB $\left.{ }^{R}\right] \mathrm{HIS}_{6}$ \\
\hline pRR05 & pGEX-4T-1 & tac & ori/Amp & GST[PCh PRP8 LexA-VP16]HIS \\
\hline pRR11 & pET & $\mathrm{T7}$ & ori/Amp & GFP[Pch PRP8 linker]FLAG \\
\hline pRR12 & $\mathrm{pET}$ & $\mathrm{T} 7$ & ori/Amp & GFP[Pch PRP8 G418 ${ }^{R}$ FLAG \\
\hline pRR13 & $\mathrm{pET}$ & $\mathrm{T} 7$ & ori/Amp & GFP[Pch PRP8 HIS5]FLAG \\
\hline pRR14 & pET & $\mathrm{T} 7$ & ori/Amp & GFP[PCh PRP8 HygB $\left.{ }^{R}\right] F L A G$ \\
\hline pRR15 & pET & $\mathrm{T} 7$ & ori/Amp & GFP[Pch PRP8 LexA-VP16]FLAG \\
\hline pRR21 & pBM258 & Gal & cen/ura3 & NLS-GFP[Pch PRP8 linker]FLAG \\
\hline pRR22 & pBM258 & Gal & cen/ura3 & NLS-GFP[PCh PRP8 G418 $]^{R}$ FLAG \\
\hline pRR23 & pBM258 & Gal & cen/ura3 & NLS-GFP[Pch PRP8 HIS5]FLAG \\
\hline pRR24 & pBM258 & Gal & cen/ura3 & NLS-GFP[Pch PRP8 HygB $\left.{ }^{R}\right] F L A G$ \\
\hline pRR25 & pBM258 & Gal & cen/ura3 & NLS-GFP[PCh PRP8 LexA-VP16]FLAG \\
\hline pRR26 & pBM258 & Gal & cen/ura3 & NLS-GFP (no intein) \\
\hline
\end{tabular}

All plasmids were constructed in this study except pGPch-1 [19]. In the table the promoter that drives expression of the extein-intein is indicated.

Episomal expression of inteins in E. coli and yeast

Expression in E. coli was performed in TunerTM (DE3) with pLysSRARE to express rare tRNAs. Bacteria were grown to early log phase in NZCYM medium supplemented with ampicillin $(100 \mu \mathrm{g} / \mathrm{ml})$ and chloramphenicol $(34 \mu \mathrm{g} / \mathrm{ml})$ then induced with $0.2 \mathrm{mM}$ isopropyl $\beta$-D-1-thiogalactopyranoside (IPTG) for $17 \mathrm{~h}$ at $20^{\circ}$. Culture densities were measured with a Klett-Summerson Colorimeter.

For plasmid-based expression in yeast from the GAL1 promoter, the strain YL $(2,4)$ LU was used with various plasmids based on pBM258. Cells were grown overnight at $30^{\circ}$ in $\mathrm{S}$-medium -ura $+2 \%$ raffinose. When cells reached a turbidity of 20 Klett units, galactose was added to a final concentration of $2 \%$ and growth was continued to 100 Klett units.

\section{Western blot analysis and the estimation of splicing efficiency}

Equivalent amounts of cells were pelleted, precipitated with $10 \%$ TCA [61], resuspended in sample buffer and separated by SDS-PAGE. Three identical gels were prepared: one directly stained by Coomassie ${ }^{\mathbb{R}}$ Brilliant Blue for detection of total protein and two transferred to Immobilon ${ }^{\mathrm{TM}}$ (Millipore, Billerica, MA) for Western blot analysis. Antibodies were either directed against GST (Santa Cruz Biotechnology, Santa Cruz, CA), Penta-His (Qiagen, Valencia, CA), GFP (Roche Applied Science, Indianapolis, IN) or the FLAG epitope (antiFLAG $^{\circledR}$ M2, Sigma-Alrich, St. Louis, MO). Antibodies were detected using Alexa Fluor 680 anti-IgG secondary (Invitrogen, Carlsbad, CA) and visualized on an Odyssey
Infrared Imaging System (LI-COR Biosciences, Lincoln, $\mathrm{NE}$ ). The Odyssey software was used to quantify band intensity. To estimate the limits of detection for the anti-GFP antibody, Western blot analysis was performed on serial dilutions of extracts from strain YL $(2,4) \mathrm{LU}$ expressing NLS-GFP from plasmid pRR26.

\section{Genomic integration of marked inteins into the CMD1 locus}

The intein cassettes were amplified from the pRR11-15 plasmids using the Expand Long Template PCR System (Roche, Indianapolis, IN). Primers included a 40 base extension homologous to the target CMD1 gene, a short linker sequence encoding L1 and L5 in Figure 4, and $\sim 23 \mathrm{bp}$ homologous to the ends of the intein sequence. The sequences of the primers were: 5'-TAGTGAATTTTTGGCTCTGATGTCTCGTCAACTCAAA TCA GGTTCAGGT AGTAAAGGAG AAGAACTTTT CACTG-3' and 5'-ATACTTTAAAAGCTTCTAGTAGTTCTTGTTCAGAGTCATT TCCAGATCC TT CGAAACCA CTGTTGTGCA G-3' in which bold sequence is homologous to the intein cassette and underlined is homologous to the CMD1 site of insertion. The amplification and integration protocols are described at the Yeast Resource Center web site http:// depts.washington.edu/yeastrc/pages/fm_3.html. KGY315 was the host except in the case of the transformation of the LexA-VP16-intein, which was transformed into YL $[6,4]$ LU.

Fluorescence microscopy was performed on a DeltaVision ${ }^{\circledR}$ imaging system (Applied Precision, Issaquah, WA) as previously reported [17]. Protein extraction and 
Western blot analysis were performed as described above using anti-GFP and anti-calmodulin antibodies [62]. The correct integration of the inteins was confirmed by four different PCR reactions using the genomic DNA as template and primer pairs from these regions: 5' UTR of CMD1 as forward primer (f.p.) $\times$ GFP as reverse primer (r.p.); GFP (f.p.) $\times$ C-terminal intein (r.p.); C-terminal intein (f.p.) $\times$ 3' UTR of CMD1(r.p.); and 5' UTR of CMD1 (f.p.) $\times$ 3' UTR of $C M D 1$ (r.p.).

\section{Acknowledgements}

We would like to thank Stefanie Pöggeler for the gift of pGPch-1 and Tzumin Lee for the gift of pBS LexA-VP16 SV40. We would like to thank Simon Diep and Jing Huang for strain YL(6,4)LU and plasmids. This work is supported by the National Center for Research Resources of the National Institutes of Health by a grant to T.N.D. entitled 'Comprehensive Biology: Exploiting the Yeast Genome', P41 RR011823.

\section{Authors' contributions}

RR carried out the plasmid constructions, molecular genetic studies and Western blot analysis, drafted the manuscript and conceived of the project. LA carried out Western blot analysis, molecular genetic studies and fluorescence microscopy. TD helped to direct the study and draft the manuscript. EM co-ordinated and directed the study, carried out fluorescence microscopy and drafted the manuscript. All authors participated in the design of the study. All authors read and approved the final manuscript.

Received: 7 March 2011 Accepted: 27 June 2011

Published: 27 June 2011

\section{References}

1. Paulus H: Protein splicing and related forms of protein autoprocessing. Annu Rev Biochem 2000, 69:447-496.

2. Perler FB: Protein splicing mechanisms and applications. IUBMB Life 2005, 57(7):469-476.

3. Hirata R, Ohsumk Y, Nakano A, Kawasaki H, Suzuki K, Anraku Y: Molecular structure of a gene, VMA1, encoding the catalytic subunit of $\mathrm{H}$ (+)-translocating adenosine triphosphatase from vacuolar membranes of Saccharomyces cerevisiae. J Biol Chem 1990, 265(12):6726-6733.

4. Kane PM, Yamashiro CT, Wolczyk DF, Neff N, Goebl M, Stevens TH: Protein splicing converts the yeast TFP1 gene product to the 69-kD subunit of the vacuolar $\mathrm{H}(+)$-adenosine triphosphatase. Science 1990, 250(4981):651-657.

5. Perler FB: InBase: the Intein Database. Nucleic Acids Res 2002, 30(1):383-384.

6. Duan X, Gimble FS, Quiocho FA: Crystal structure of PI-Scel, a homing endonuclease with protein splicing activity. Cell 1997, 89(4):555-564

7. Chong S, Shao Y, Paulus H, Benner J, Perler FB, XU MQ: Protein splicing involving the Saccharomyces cerevisiae VMA intein. The steps in the splicing pathway, side reactions leading to protein cleavage, and establishment of an in vitro splicing system. J Biol Chem 1996, 271(36):22159-22168.

8. Derbyshire V, Wood DW, Wu W, Dansereau JT, Dalgaard JZ, Belfort M: Genetic definition of a protein-splicing domain: functional mini-inteins support structure predictions and a model for intein evolution. Proc Natl Acad Sci USA 1997, 94(21):11466-11471.

9. Elleuche S, Doring K, Poggeler S: Minimization of a eukaryotic mini-intein. Biochem Biophys Res Commun 2008, 366(1):239-243.

10. Cheriyan M, Perler FB: Protein splicing: A versatile tool for drug discovery. Adv Drug Deliv Rev 2009, 61(11):899-907.

11. Elleuche S, Poggeler S: Inteins, valuable genetic elements in molecular biology and biotechnology. Appl Microbiol Biotechnol 2010, 87(2):479-489.

12. Vila-Perello M, Muir TW: Biological applications of protein splicing. Cell 2010, 143(2):191-200
13. Muralidharan V, Muir TW: Protein ligation: an enabling technology for the biophysical analysis of proteins. Nature methods 2006, 3(6):429-438.

14. Amitai G, Callahan BP, Stanger MJ, Belfort G, Belfort M: Modulation of intein activity by its neighboring extein substrates. Proc Natl Acad Sci USA 2009, 106(27):11005-11010

15. Kollman JM, Zelter A, Muller EG, Fox B, Rice LM, Davis TN, Agard DA: The structure of the gamma-tubulin small complex: implications of its architecture and flexibility for microtubule nucleation. Mol Biol Cell 2008, 19(1):207-215

16. Mc Intyre J, Muller EG, Weitzer S, Snydsman BE, Davis TN, Uhlmann F: In vivo analysis of cohesin architecture using FRET in the budding yeast Saccharomyces cerevisiae. EMBO J 2007, 26(16):3783-3793.

17. Muller EG, Snydsman BE, Novik I, Hailey DW, Gestaut DR, Niemann CA, O'Toole ET, Giddings TH, Sundin BA, Davis TN: The organization of the core proteins of the yeast spindle pole body. Mol Biol Cell 2005, 16(7):3341-3352.

18. Mathieson EM, Suda Y, Nickas M, Snydsman B, Davis TN, Muller EG, Neiman AM: Vesicle docking to the spindle pole body is necessary to recruit the exocyst during membrane formation in Saccharomyces cerevisiae. Molecular biology of the cell 2010, 21(21):3693-3707.

19. Elleuche S, Nolting N, Poggeler S: Protein splicing of PRP8 mini-inteins from species of the genus Penicillium. Appl Microbiol Biotechnol 2006, 72(5):959-967.

20. Elleuche S, Poggeler S: Trans-splicing of an artificially split fungal miniintein. Biochem Biophys Res Commun 2007, 355(3):830-834.

21. Huang J, Schreiber SL: A yeast genetic system for selecting small molecule inhibitors of protein-protein interactions in nanodroplets. Proc Natl Acad Sci USA 1997, 94(25):13396-13401.

22. Nurizzo D, Shewry SC, Perlin MH, Brown SA, Dholakia JN, Fuchs RL, Deva T, Baker EN, Smith CA: The crystal structure of aminoglycoside-3'phosphotransferase-lla, an enzyme responsible for antibiotic resistance. $J$ Mol Biol 2003, 327(2):491-506.

23. Glynn SE, Baker PJ, Sedelnikova SE, Davies CL, Eadsforth TC, Levy CW, Rodgers HF, Blackburn GM, Hawkes TR, Viner R, Rice DW: Structure and mechanism of imidazoleglycerol-phosphate dehydratase. Structure 2005 13(12):1809-1817.

24. Stogios PJ, Shakya T, Evdokimova E, Savchenko A, Wright GD: Structure and function of $\mathrm{APH}(4)-\mathrm{la}$, a hygromycin B resistance enzyme. The Journal of biological chemistry 2011, 286(3):1966-1975.

25. Fogh RH, Ottleben G, Ruterjans H, Schnarr M, Boelens R, Kaptein R: Solution structure of the LexA repressor DNA binding domain determined by $1 \mathrm{H}$ NMR spectroscopy. The EMBO journal 1994, 13(17):3936-3944.

26. Davis TN, Urdea MS, Masiarz FR, Thorner J: Isolation of the yeast calmodulin gene: calmodulin is an essential protein. Cell 1986, 47(3):423-431

27. Brockerhoff SE, Davis TN: Calmodulin concentrates at regions of cell growth in Saccharomyces cerevisiae. J Cell Biol 1992, 118(3):619-629.

28. Huh WK, Falvo JV, Gerke LC, Carroll AS, Howson RW, Weissman JS, O'Shea EK: Global analysis of protein localization in budding yeast. Nature 2003, 425(6959):686-691.

29. Riffle M, Davis TN: The Yeast Resource Center Public Image Repository: A large database of fluorescence microscopy images. BMC bioinformatics 2010, 11:263.

30. Flavell RR, Muir TW: Expressed protein ligation (EPL) in the study of signal transduction, ion conduction, and chromatin biology. Acc Chem Res 2009, 42(1):107-116.

31. Adam E, Perler FB: Development of a positive genetic selection system for inhibition of protein splicing using mycobacterial inteins in Escherichia coli DNA gyrase subunit A. J Mol Microbiol Biotechnol 2002, 4(5):479-487.

32. Zeidler MP, Tan C, Bellaiche Y, Cherry S, Hader S, Gayko U, Perrimon N: Temperature-sensitive control of protein activity by conditionally splicing inteins. Nat Biotechnol 2004, 22(7):871-876.

33. Tan G, Chen M, Foote C, Tan C: Temperature-sensitive mutations made easy: generating conditional mutations by using temperature-sensitive inteins that function within different temperature ranges. Genetics 2009, 183(1):13-22.

34. Tyszkiewicz AB, Muir TW: Activation of protein splicing with light in yeast. Nat Methods 2008, 5(4):303-305. 
35. Buskirk AR, Ong YC, Gartner ZJ, Liu DR: Directed evolution of ligand dependence: small-molecule-activated protein splicing. Proc Natl Acad Sci USA 2004, 101(29):10505-10510.

36. Mootz HD, Blum ES, Tyszkiewicz AB, Muir TW: Conditional protein splicing: a new tool to control protein structure and function in vitro and in vivo. J Am Chem Soc 2003, 125(35):10561-10569.

37. Mootz HD, Muir TW: Protein splicing triggered by a small molecule. J Am Chem Soc 2002, 124(31):9044-9045.

38. Theodoro RC, Volkmann G, Liu XQ, Bagagli E: PRP8 intein in Ajellomycetaceae family pathogens: Sequence analysis, splicing evaluation and homing endonuclease activity. Fungal Genet Biol 2010.

39. Elleuche S, Pelikan C, Nolting N, Poggeler S: Inteins and introns within the prp8 -gene of four Eupenicillium species. J Basic Microbiol 2009, 49(1):52-57.

40. Butler MI, Gray J, Goodwin TJ, Poulter RT: The distribution and evolutionary history of the PRP8 intein. BMC Evol Biol 2006, 6:42.

41. Wach A, Brachat A, Alberti-Segui C, Rebischung C, Philippsen P: Heterologous HIS3 marker and GFP reporter modules for PCR-targeting in Saccharomyces cerevisiae. Yeast 1997, 13(11):1065-1075.

42. Ding $Y, X u$ MQ, Ghosh I, Chen X, Ferrandon S, Lesage G, Rao Z: Crystal structure of a mini-intein reveals a conserved catalytic module involved in side chain cyclization of asparagine during protein splicing. The Journal of biological chemistry 2003, 278(40):39133-39142.

43. Hall TM, Porter JA, Young KE, Koonin EV, Beachy PA, Leahy DJ: Crystal structure of a Hedgehog autoprocessing domain: homology between Hedgehog and self-splicing proteins. Cell 1997, 91(1):85-97.

44. Klabunde T, Sharma S, Telenti A, Jacobs WR, Sacchettini JC: Crystal structure of GyrA intein from Mycobacterium xenopi reveals structural basis of protein splicing. Nature structural biology 1998, 5(1):31-36.

45. Koonin EV: A protein splice-junction motif in hedgehog family proteins. Trends Biochem Sci 1995, 20(4):141-142.

46. Perler FB: Protein splicing of inteins and hedgehog autoproteolysis: structure, function, and evolution. Cell 1998, 92(1):1-4

47. Sun P, Ye S, Ferrandon S, Evans TC, Xu MQ, Rao Z: Crystal structures of an intein from the split dnaE gene of Synechocystis sp. PCC6803 reveal the catalytic model without the penultimate histidine and the mechanism of zinc ion inhibition of protein splicing. Journal of molecular biology 2005, 353(5):1093-1105.

48. Van Roey P, Pereira B, Li Z, Hiraga K, Belfort M, Derbyshire V: Crystallographic and mutational studies of Mycobacterium tuberculosis recA mini-inteins suggest a pivotal role for a highly conserved aspartate residue. Journal of molecular biology 2007, 367(1):162-173.

49. Krishna MM, Englander SW: The N-terminal to C-terminal motif in protein folding and function. Proc Natl Acad Sci USA 2005, 102(4):1053-1058.

50. Mounce BC, Kurt N, Ellison PA, Cavagnero S: Nonrandom distribution of intramolecular contacts in native single-domain proteins. Proteins 2009, 75(2):404-412.

51. Jacob $E$, Unger $R$ : $A$ tale of two tails: why are terminal residues of proteins exposed? Bioinformatics 2007, 23(2):e225-230.

52. Prein $\mathrm{B}$, Natter $\mathrm{K}$, Kohlwein SD: A novel strategy for constructing $\mathrm{N}$ terminal chromosomal fusions to green fluorescent protein in the yeast Saccharomyces cerevisiae. FEBS Lett 2000, 485(1):29-34.

53. Gauss R, Trautwein M, Sommer T, Spang A: New modules for the repeated internal and $\mathrm{N}$-terminal epitope tagging of genes in Saccharomyces cerevisiae. Yeast 2005, 22(1):1-12.

54. Schneider BL, Seufert W, Steiner B, Yang QH, Futcher AB: Use of polymerase chain reaction epitope tagging for protein tagging in Saccharomyces cerevisiae. Yeast 1995, 11(13):1265-1274.

55. Greenland KB, Ding H, Costanzo M, Boone C, Davis TN: Identification of Saccharomyces cerevisiae Spindle Pole Body Remodeling Factors. PLOS One 2010, 5(11):e15426.

56. Wallis JW, Chrebet G, Brodsky G, Rolfe M, Rothstein R: A hyperrecombination mutation in $\mathrm{S}$. cerevisiae identifies a novel eukaryotic topoisomerase. Cell 1989, 58(2):409-419.

57. [http://depts.washington.edu/yeastrc/].

58. Lai SL, Lee T: Genetic mosaic with dual binary transcriptional systems in Drosophila. Nat Neurosci 2006, 9(5):703-709.

59. Johnston M, Davis RW: Sequences that regulate the divergent GAL1GAL10 promoter in Saccharomyces cerevisiae. Mol Cell Biol 1984, 4(8):1440-1448.
60. Benson DA, Karsch-Mizrachi I, Lipman DJ, Ostell J, Sayers EW: GenBank. Nucleic Acids Res 2010

61. Wright AP, Bruns M, Hartley BS: Extraction and rapid inactivation of proteins from Saccharomyces cerevisiae by trichloroacetic acid precipitation. Yeast 1989, 5(1):51-53

62. Moser MJ, Lee SY, Klevit RE, Davis TN: Ca2+ binding to calmodulin and its role in Schizosaccharomyces pombe as revealed by mutagenesis and NMR spectroscopy. J Biol Chem 1995, 270(35):20643-20652.

doi:10.1186/1472-6750-11-71

Cite this article as: Ramsden et al:: An intein with genetically selectable markers provides a new approach to internally label proteins with GFP. BMC Biotechnology 2011 11:71.

\section{Submit your next manuscript to BioMed Central and take full advantage of:}

- Convenient online submission

- Thorough peer review

- No space constraints or color figure charges

- Immediate publication on acceptance

- Inclusion in PubMed, CAS, Scopus and Google Scholar

- Research which is freely available for redistribution

Submit your manuscript at www.biomedcentral.com/submit
C) Biomed Central 